\title{
ON EXPANSIVE TRANSFORMATION GROUPS
}

\author{
BY \\ PING-FUN LAM
}

1. Introduction. Expansive transformation groups and its special case-expansive homeomorphisms have been studied by B. F. Bryant, M. Eisenberg, W. H. Gottschalk, G. A. Hedlund, E. Hemmingsen, J. F. Jakobsen, H. Keynes, W. Reddy, S. Schwartzman, W. Utz, R. F. Williams and T. S. Wu et al. The shift of a symbolic flow is an expansive homeomorphism. A typical example of an expansive homeomorphism acting on a manifold is any member of a unimodular group without eigenvalues of absolute value 1 acting in the usual way on a $n$-dimensional torus $(n \geqq 2)$. The latter is also an expansive automorphism acting on a compact group. The shift of a $p$-adic solenoid is another such automorphism. In this paper we obtain some partial results on the following two problems: (A) to characterize and to classify all uniform spaces which admit an expansive homeomorphism or a positively expansive map, (B) to characterize and, to classify all topological groups which admit an expansive automorphism or an expansive endomorphism. Among other results we give an affirmative answer to the following better known open question.

QUESTION. If a compact connected group admits an expansive automorphism, must it be abelian?

We obtain, in fact, a much more general result (cf. Theorem 3.2). The question was raised in [4], where the case for compact connected Lie groups was also answered affirmatively. The case for finite-dimensional compact connected groups was proved by Wu [17]. Using Wu's result, Eisenberg [6] shows also that if a compact connected finite-dimensional group admits a surjective expansive endomorphism, then it is abelian. Theorem 3.2 includes all these results. In Theorem 3.5 we slightly generalize Theorem 3.2 from compact groups to maximally almost periodic groups. The other results that we have are, for most part, improvement of the previously known results on (A).

\section{Expansive family of continuous maps on uniform spaces.}

2.1. Definition. Let $X$. be a uniform space with uniformity $\mathscr{U}$. A nonempty family of continuous maps, $\mathscr{F}$, of $X$ inta $X$ is said to be expansive, if there exists $\alpha \in \mathscr{U}$ such that for every $x, y \in X, x \neq y$, there is $f \in \mathscr{F}$, depending on $x$ and $y$ so that $(f(x), f(y)) \notin \alpha$. The uniform index $\alpha$, which is not unique, is then called an expansive index of $\mathscr{F}$.

Received by the editors December 6, 1968. 
It follows easily that if $X$ admits such $\mathscr{F}$ then $X$ is $T_{2}$.

2.2. Definition. Let $(X, \mathscr{U})$ be a uniform space. A transformation group or transformation semigroup $(X, T, \pi)$ is said to be expansive if the family $\mathscr{F}=$ $\left\{\pi^{t} \mid t \in T\right\}$ is expansive on $X$. A self-homeomorphism $h$ of $X$ is said to be expansive if the discrete flow generated by $h$ is expansive on $X$, i.e. iff the family $\mathscr{F}_{1}=$ $\left\{h^{n} \mid n=0, \pm 1, \ldots\right\}$ is expansive. A continuous map $f$ of $X$ into $X$ is said to be (positively) expansive if the semigroup $\mathscr{F}_{2}=\left\{f^{n} \mid n=0,1,2, \ldots\right\}$ generated by $f$ is expansive.

Our first consideration is the metrizability of such a space $X$. First we state the latest known result in this respect.

2.3. TheOREM (BRyANt-EISENBERG). If a compact uniform space $X$ admits an expansive transformation semigroup $(X, T, \pi)$, where $T$ is separable, then $X$ is metrizable.

The theorem was first proved by Bryant [3] for the case of expansive homeomorphism and then generalized by Eisenberg [5].

In the following we will consider spaces which are not necessarily compact.

2.4. Lemma. If a uniform space ( $X, \mathbb{I})$ admits a nonempty countable expansive family of continuous maps $\mathscr{F}$, then every compact subset of $X$ is metrizable.

Proof. Let $K$ be an arbitrary compact subset of $X, \mathscr{U}_{K}$ denote the induced uniformity of $\mathscr{U}$ on $K$, and $\mathscr{F}=\left\{f_{1}, f_{2}, f_{3}, \ldots\right\}$. Let $\hat{f}_{n}: X \times X \rightarrow X \times X$ be defined as follows

$$
\hat{f}_{n}(x, y)=\left(f_{n}(x), f_{n}(y)\right) ; \quad x, y \in X, \quad n \text { integers. }
$$

Let $\alpha$ be an expansive index of $\mathscr{F}$. We may assume $\alpha$ to be closed. Let $\alpha_{n}=\hat{f}_{n}^{-1}(\alpha)$ and $\alpha_{n}(K)=\alpha_{n} \cap(K \times K)$. Then $\alpha_{n}(K) \in \mathscr{U}_{K}$. Define $\beta_{n}(K)=\bigcap_{i=1}^{n} \alpha_{i}(K) \in \mathscr{U}_{K}$. To prove the lemma it suffices to show that $\beta_{1}(K), \beta_{2}(K), \ldots$ form a base for $\mathscr{U}_{K}$. Thus let $\gamma(K)$ be an open index of $\mathscr{U}_{K}$. By the expansiveness of $\mathscr{F}$, it follows that

$$
(K \times K-\gamma(K)) \cap\left(\bigcap_{n=1}^{\infty} \alpha_{n}(K)\right)=\varnothing .
$$

Since $K$ is compact, there exists a positive integer $N$ such that

$$
(K \times K-\gamma(K)) \cap\left(\bigcap_{n=1}^{N} \alpha_{n}(K)\right)=\varnothing .
$$

Hence $\beta_{N}(K) \subset \gamma(K)$ and this proves the lemma.

The following statement is obvious.

2.5. Lemma. Let $\mathscr{C}$, the set of all continuous maps of uniform space $X$ into $X$, be given the pointwise-convergence topology and $\varnothing \neq \mathscr{F} \subset \mathscr{C}$. Then $\mathscr{F}$ is expansive on $X$ iff $\mathscr{F}$ is expansive on $X$.

2.6. Theorem (Ju. M. SMIRnov [14]). A locally metrizable $T_{2}$ space is metrizable iff it is paracompact. 
2.7. THEOREM. Let $X$ be a uniform space which is locally compact and paracompact. Let $\mathscr{C}$, the set of all continuous maps of $X$ into $X$, be given the pointwiseconvergence topology and $\varnothing \neq \mathscr{F} \subset \mathscr{C}$. If $\mathscr{F}$ is separable and expansive on $X$, then $X$ is metrizable.

Proof. Use 2.4, 2.5, and 2.6.

2.8. Corollary. Let $X$ be a uniform space which is locally compact and paracompact. If $X$ admits an expansive transformation semigroup $(X, T, \pi)$, where $T$ is separable, then $X$ is metrizable.

In the remainder of the section, we restrict our attention to expansive homeomorphisms. We first prove a theorem which gives a canonical form for an expansive homeomorphism on a compact uniform space $X$. Note that $X$ is necessarily metrizable. First some notations:

$I=[0,1]$, the unit interval,

$Z=$ the set of integers,

$\Omega=I^{Z}$, with product topology, i.e. $\Omega$ is the Hilbert cube,

$\sigma=$ the shift of $\Omega ; \sigma(x)_{i}=x_{i+1}, x \in \Omega$.

2.9. TheOREM. For every expansive homeomorphism $h$ on a compact metric space $X$, there exist a positive integer $n$ and a subset $Y$ of $\Omega$ which is invariant under $\sigma^{n}$ such that $(X, h)$ and $\left(Y, \sigma^{n}\right)$ are isomorphic as dynamical systems.

Proof. Let $d$ be the metric on $X$. By the expansiveness of $h$, there exists a number $c>0$, so called an expansive constant, such that if $x, y \in X, x \neq y$, then $d\left(h^{n}(x), h^{n}(y)\right)$ $>c$ for some integer $n$. Let $S_{r}(x)$ denote the open $r$-ball of a point $x \in \in^{\prime} X$, where $r>0$. For $x \in X$, by the completely regularity of $X$, there exists a continuous function $f_{x}: X \rightarrow I$ whose support lies in $S_{c / 2}(x)$ and $f_{x}(x)=1$. Let

$$
V_{x}=\left\{y \in X \mid f_{x}(y) \neq 0\right\} .
$$

Then there exists $x_{0}, x_{1}, \ldots, x_{n-1} \in X$ such that $X=\bigcup_{i=0}^{n-1} V_{x_{i}}$. Define maps $g_{i, k}=f_{x_{i}} \circ h^{k}: X \rightarrow I$, where $0 \leqq i \leqq n-1$ and $k$ runs over all integers. By the expansiveness of $h$, the family $\left\{g_{i, k} \mid 0 \leqq i \leqq n-1, k \in Z\right\}$ separate points of $X$. Let $\varphi: X \rightarrow \Omega$ be defined as follows

$$
\varphi(x)_{k n+i}=g_{i, k}(x) ; \quad x \in X, \quad k \in Z, \quad 0 \leqq i \leqq n-1 .
$$

Then $\varphi$ is continuous and injective, whence it is a homeomorphism of $X$ onto $\varphi(X) \subset \Omega$. Furthermore

$$
\varphi(h(x))_{k n+i}=g_{i, k}(h(x))=g_{i, k+1}(x)=\varphi(x)_{k n+i+n}=\left[\sigma^{n}(\varphi(x))\right]_{k n+i}
$$

Hence

$$
\varphi(h(x))=\sigma^{n}(\varphi(x)), \quad x \in X .
$$

Let $Y=\varphi(X)$, then $Y$ is invariant under $\sigma^{n}, \sigma^{n}(Y)=Y$. Also $\varphi$ is an isomorphism of $(X, h)$ onto $\left(Y, \sigma^{n}\right)$ in the sense of dynamical system. 
The next theorem concerns the number of nonwandering points of a space $X$ which has an expansive homeomorphism.

2.10. Lemma. Let $X$ be a $\sigma$-compact uniform space which admits an expansive homeomorphism $h$. Let $a, b$ be fixed points of $h$ which are not necessarily distinct. Then the set

$$
M_{a, b}=\left\{x \in X \mid \lim _{n \rightarrow \infty} h^{n}(x)=a, \lim _{n \rightarrow-\infty} h^{n}(x)=b\right\}
$$

is at most countable.

Proof. When $X$ is compact, this result is due to Reddy [13]. Now let $X=\bigcup_{i=1}^{\infty} K_{i}$, $K_{i}$ compact. If $M_{a, b}$ is uncountable, then $M_{a, b} \cap K_{n}$ is uncountable for some $n$. The proof of the given reference is easily extended to obtain a contradiction for our case.

2.11. TheOREM. Let $X$ be an uncountable $\sigma$-compact metric space. If $h$ is an expansive homeomorphism on $X$ such that every orbit has compact closure, then the set of nonwandering points of $h$ in $X$ is infinite.

Proof. Let $\Omega$ denote the set of nonwandering points. This set is invariant under $h$. Suppose the contrast that $\Omega$ has at most finite number of points. Then there exists a positive integer $n$ such that $\left.h^{n}\right|_{\Omega}$ is the identity map. We let $g=h^{n}$, then $g$ is also expansive on $X$, and it is easy to see that $\Omega$ remains to be the set of nonwandering points of $g$. Let $x \in X$. We claim $\omega(x) \subset \Omega$, where $\omega(x)$ is the $\omega$-limiting points of $x$. For if there is $y \in \omega(x)-\Omega$, then for every neighborhood $U$ of $y$ there exists integers $m, k$ with $m-k$ arbitrary large so that $g^{m}(x), g^{k}(x) \in U$, which implies $g^{m-k}(U) \cap U \neq \varnothing$, whence contradicts that $y \notin \Omega$. Likewise we can easily show $\alpha(x) \subset \Omega$, where $\alpha(x)$ is the $\alpha$-limiting set of $x$. Since the orbit $O(x)$ has compact closure, we have $\alpha(x) \neq \varnothing \neq \omega(x)$. We conclude first $\Omega \neq \varnothing$. Now if $y \in \omega(x)$, then $\lim _{n \rightarrow \infty} g^{n}(x)=y$. Otherwise, using the facts that $\mathrm{cl}(O(x))$ is compact and $y$ is a fixed point of $g$, we easily obtain points $p \in \Omega$ arbitrary near $y$, which contradicts that $\Omega$ is finite. Likewise we have $\lim _{n \rightarrow-\infty} g^{n}(x)$ exists for all $x \in X$. Hence

$$
X=\bigcup\left\{M_{a, b} \mid(a, b) \in \Omega \times \Omega\right\},
$$

where $M_{a, b}$ is defined as in the lemma with $g$ in place of $h$. Since $X$ is uncountable, so must be one of the $M_{a, b}$. This contradicts the lemma.

2.12. Corollary. Let $X$ be an uncountable compact metric space, and $h$ an expansive homeomorphism on $X$. Then the set of nonwandering points of $X$ is infinite.

Existence theorems for expansive homeomorphisms are often very interesting. For instance, it was shown in [2] that there is no expansive homeomorphism on an arc and in [11] the same is true for a circle. It follows that there is no expansive homeomorphisms on a compact graph which has at least one edge or on any 2-manifold whose boundary is compact and nonempty. The question as to whether 
there is any expansive homeomorphism on the 2-sphere remains open. By taking the square of the homeomorphism, we can reduce the problem to the case where the homeomorphism is orientation preserving, whence it has a fixed point. In the following we show that the homeomorphism can be expansive only if it has more than one fixed points. On the other hand it can easily be shown that an expansive homeomorphism has only finite number of fixed points. Hence our partial result would solve the problem if somehow we have a way to reduce the number of fixed points.

2.13. THEOREM. There is no orientation preserving expansive homeomorphism on the 2-sphere with exactly one fixed point.

Proof. It follows, essentially, from the Brouwer's translation theorem that any orientation preserving homeomorphism on the 2-sphere has exactly one nonwandering point (cf. [1]). 2.12 then gives the theorem. We remark that by using another form of the Brouwer's translation theorem, 2.13 can also be obtained from 2.10.

3. Expansive family of endomorphisms on topological groups. The definitions and results of $\$ 2$ apply to the special case when the phase space $X$ is a topological group $G$. For this case the paracompactness condition of 2.7 can be omitted. This is due to the fact that, by the Birkhoff-Kakutani theorem, a locally metrizable group is metrizable. The uniformity we often use and we will use for $G$ is its left or right uniformity. It is easy to see that a family of (continuous) endomorphisms $\mathscr{F}$ of $G$ into $G$ is expansive with respect to one of the two uniformities iff it is so to the other. For either case the expansiveness is equivalent to the existence of a neighborhood $V$ of the identity $e \in G$ such that if $x \in G, x \neq e$, then there is $f \in \mathscr{F}$ so that $f(x) \notin V$. It turns out also that the groups we are going to study have equal left and right uniformities.

We now state and prove the theorem which answers the question stated in the introduction. The author is much in debt to Professor G. D. Mostow, who pointed out the following fact to me by conversation.

3.1. RemarK. Let $G$ be a compact connected semisimple Lie group. Let $\mathscr{E}$, the set of all endomorphisms of $G$ into $G$, be given the compact-open topology. Then $\mathscr{E}$ is compact.

3.2. THeOREM. Let $G$ be a compact connected group. If $G$ admits a nonempty expansive family of endomorphisms, then $G$ is abelian.

Proof. Let $\mathscr{F}=\left\{f_{\alpha}\right\}_{\alpha}$ be the family of expansive endomorphisms of $G$. Corresponding to it there is then a neighborhood $V$ of the identity $e \in G$ such that, if $x \in G, x \neq e$ then there exists some $f_{\alpha} \in \mathscr{F}$ so that $f_{\alpha}(x) \notin V$. Let $U$ be a neighborhood of $e$ such that $U^{2} \subset V$. There exists a compact normal subgroup $K, K \subset U$ and $G^{*}=G / K$ is a compact connected Lie group. We assert that $G^{*}$ is abelian. Thus let $\pi: G \rightarrow G^{*}$ be the canonical projection. There exists an injective local homomorphism $\varphi$ of $G^{*}$ into $G$ whose inverse map is, in fact, $\pi$. This assigns every 
$f_{\alpha} \in \mathscr{F}$ a local homomorphism $g_{\alpha}$ of $G^{*}$ to $G^{*}$ by $g_{\alpha}=\pi \circ f_{\alpha} \circ \varphi$. Set $\mathscr{G}=\left\{g_{\alpha}\right\}_{\alpha}$. Suppose $G^{*}$ is not abelian. Then $H$, the commutator subgroup of $G^{*}$ is nontrivial. Note that $H$ is compact, connected and semisimple. Let $\mathscr{L}\left(G^{*}\right)$ denote the Lie algebra of $G^{*}$. Its derived algebra $\left[\mathscr{L}\left(G^{*}\right), \mathscr{L}\left(G^{*}\right)\right]$ then coincides with $\mathscr{L}(H)$, the Lie algebra of $H$. Let $W$ be the domain of $\varphi$, which we may assume to be open and connected. Then

(i) For every $a \in W-\{e\}$ there exists $f_{\beta} \in \mathscr{F}$ such that $g_{\beta}(a) \cap \pi(U)=\varnothing$. This follows easily from the expansiveness of $\mathscr{F}$, the injective property of $\varphi$ and the choices of $U$ and $K$. We fix a metric on $G$. Let $S_{r}(r>0)$ denote the $r$-open ball of $e \in G^{*}$. Each $S_{r}$ then contains a nondegenerated subset $A_{r}$ of $H$ such that $e \in A_{r}$. Then from (i) we have

(ii) For each triple of sufficiently small numbers $r, s, t, 0<t<s<r$, there exists some $g_{\alpha}^{r, s, t} \in \mathscr{G}$ such that

$$
g_{\alpha}^{r, s, t}\left(A_{t}\right) \cap\left(\bar{S}_{r}-S_{s}\right) \neq \varnothing .
$$

Now each $g_{\alpha} \in \mathscr{G}$ induces a derivative $d g_{\alpha}: \mathscr{L}\left(G^{*}\right) \rightarrow \mathscr{L}\left(G^{*}\right)$, which is a (Lie) homomorphism. We then have

(iii) (a) $d g_{\alpha}(\mathscr{L}(H)) \subset \mathscr{L}(H)$.

(b) For a small neighborhood $N$ of $0 \in \mathscr{L}\left(G^{*}\right)$ we have

$$
g_{\alpha} \circ \exp =\exp \circ d g_{\alpha}, \quad g_{\alpha} \in \mathscr{G},
$$

where, of course, exp is the exponential map of $\mathscr{L}\left(G^{*}\right)$ onto $G^{*}$. Now in (ii) we fix a pair of small $r$ and $s$ and let $t$ tend to 0 . Using (iii) we then have a neighborhood $P$ of $0 \in \mathscr{L}\left(G^{*}\right)$, a sequence $\left\{y_{i}\right\} \subset \mathscr{L}(H)-\{0\}$, a sequence $\left\{d g_{\alpha_{i}}\right\}$ such that

$$
\lim _{i \rightarrow \infty} y_{i}=0 \text { and } d g_{\alpha_{i}}\left(y_{i}\right) \notin P .
$$

Let $\tilde{H}$ denote the universal covering group of $H$, whose identity we denote as $e_{1}$. Note that $\tilde{H}$ is a compact, simply-connected and semisimple Lie group. Each $d g_{\alpha_{||} \mathscr{L}_{(H)}}$ induces an endomorphism $h_{\alpha_{i}}$ of $\tilde{H}$ whose derivative is $d g_{\alpha_{i \mid} \mathscr{L}_{(H)}}$ itself. We then have a neighborhood $Q$ of $e_{1}$, a sequence $\left\{b_{i}\right\} \subset \tilde{H}-\left\{e_{1}\right\}$, such that

$$
\lim _{i \rightarrow \infty} b_{i}=e_{1} \quad \text { and } \quad h_{\alpha_{i}}\left(b_{i}\right) \notin Q .
$$

However, by $3.1, \mathscr{E}(\tilde{H})$, the set of all endomorphisms of $\tilde{H}$ is compact with the compact-open topology. In particular the family $\left\{h_{\alpha_{t}} \mid i=1,2, \ldots\right\}$ should be equicontinuous at $e_{1}$. We then have a contradiction. The contradiction shows our assertion, that $G^{*}$ is abelian. Since $K$ can be chosen to be arbitrary small, the group $G$ itself must be abelian. This completes the proof of the theorem.

3.3. Corollary. Let $G$ be a compact connected group. If $G$ admits an expansive automorphism \{endomorphism\}, then $G$ is abelian.

3.4. Remark. (1) The connectedness of $G$ in 3.2 and 3.3 is essential, for the identity map of any finite discrete group is expansive. (2) If $G$ is only locally com- 
pact and connected, then 3.2 and 3.3 do not hold in general. In fact there is a Lie group, which is topologically the Euclidean 3-space, which admits an expansive automorphism and the group is not abelian (cf. [5]). (3) The existence of expansive automorphisms on an infinite-dimensional compact connected group remains to be an open question. But it is easy to construct an expansive group of automorphisms with two commuting generators on the countable product of the circle group.

In spite of the difficulty indicated in (2) of 3.4, Theorem 3.2 can be somewhat generalized.

3.5. THEOREM. Let $G$ be a locally compact, connected, maximally almost periodic group. If $G$ admits an expansive $(\neq \varnothing)$ family $\mathscr{F}$ of endomorphisms, then $G$ is abelian.

Proof. By the Freudenthal-Weil theorem (cf. [10, p. 50])

$$
G=K \times V \quad \text { (as topological groups), }
$$

where $K$ is compact and $V$ is a vector group. Clearly then $K$ contains all compact subgroups of $G$. Hence, if $f \in \mathscr{F}$ then $f(K) \subset K$. Also $K$ must be connected. By 3.2, we conclude that $K$ is abelian. Hence $G$ is abelian.

Finally we give a canonical form for an expansive automorphism of a compact connected group. Let $K$ be the circle group, $Z$ the set of integers, then $K^{Z}$ is the infinite toroid group. Let $\sigma$ denote its shift.

3.6. THEOREM. Let $h$ be an expansive automorphism on a compact connected group $G$. Then there exists a positive integer $n$, a compact subgroup $H$ of $K^{Z}$ which is invariant under $\sigma^{n}$ and a map $\varphi$ of $G$ onto $H$ which is both a topological group isomorphism and an isomorphism of $(G, h)$ to $\left(H, \sigma^{n}\right)$ in the sense of dynamical systems.

Proof. From 2.7 and 3.2 we know $G$ is a metrizable compact abelian group. Let $\hat{G}$ denote the character group of $G$, then $\hat{G}$ is countable. We let

$$
\hat{G}=\left\{\chi_{i} \mid i=0, \pm 1, \pm 2, \ldots\right\} \text {. }
$$

A topological group isomorphism $\pi$ of $G$ into $K^{Z}$ can, therefore, be defined as follows. Let $x \in G$, then

$$
\pi(x)_{i}=\chi_{i}(x), \quad i \in Z .
$$

Let $g$ be the automorphism of $\pi(G)$ given by $g=\pi \circ h \circ \pi^{-1}$. Then $g$ is expansive on $\pi(G)$. Let $p_{i}: K^{Z} \rightarrow K$ denote the $i$ th-projection, $i \in Z$. By the expansiveness of $g$, there exists a neighborhood $V$ of the identity of $K$ and a positive integer $n_{1}$ such that, if $y \in \pi(G)$ is not the identity then there is some integer $m$ so that

$$
g^{m}(y) \notin \bigcap_{i=-n_{1}}^{n_{1}} p_{i}^{-1}(V) \text {. }
$$

Now define maps $f_{k, i}=p_{i-n_{1}} \circ g^{k}: \pi(G) \rightarrow K$, where $0 \leqq i \leqq 2 n_{1}$ and $k$ runs through all integers. Then $\left\{f_{k, i} \mid 0 \leqq i \leqq 2 n_{1}, k \in Z\right\} \subset(\pi(G))^{\wedge}$, the character group of $\pi(G)$. 
Note that $\left\{f_{k, i} \mid 0 \leqq i \leqq 2 n, k \in Z\right\}$ separate points of $\pi(G)$. We can then define an isomorphism $\psi$ of $\pi(G)$ into $K^{Z}$ as follows. Let $n=2 n_{1}+1, y \in \pi(G)$. By definition

$$
\psi(y)_{k n+i}=f_{k, i}(y), \quad k \in Z, \quad 0 \leqq i<n .
$$

Then $\psi(g(y))=\sigma^{n}(\psi(y)), y \in \pi(G)$. Let $H=\psi(\pi(G))$, then $H$ is invariant under $\sigma^{n}$. Let $\varphi=\psi \circ \pi$. Then $\varphi$ is a topological group isomorphism of $G$ onto $H$ and

$$
\varphi \circ h=\psi \circ \pi \circ h=\psi \circ g \circ \pi=\sigma^{n} \circ \psi \circ \pi=\sigma^{n} \circ \varphi .
$$

This completes the proof of the theorem.

\section{REFERENCES}

1. S. A. Andrea, On homeomorphisms of the plane, and their embedding in flows, Bull. Amer. Math. Soc. 71 (1965), 381-383. MR 30 \#2478.

-2. B. F. Bryant, Expansive self-homeomorphisms of a compact metric space, Amer. Math. Monthly 69 (1962), 386-391.

3. - On expansive homeomorphisms, Pacific J. Math. 10 (1960), 1163-1167. MR 22 \#11382.

4. M. Eisenberg, Expansive transformation semigroups of endomorphisms of topological groups, Dissertation, Wesleyan University, Middletown, Conn., 1965.

5. - Expansive transformation semigroups of endomorphisms, Fund. Math. 59 (1966), 313-321.

6. - A note on positively expansive endomorphisms, Math. Scand. 19 (1966), 217-218.

7. W. H. Gottschalk and G. A. Hedlund, Topological dynamics, Amer. Math. Soc. Colloq. Publ., vol. 36, Amer. Math. Soc., Providence, R. I., 1955. MR 17, 650.

8. G. A. Hedlund and M. Morse, Symbolic dynamics, Amer. J. Math. 60 (1938), 815-866.

9. G. Hochschild, The structure of Lie groups, Holden-Day, San Francisco, Calif., 1965. MR 34 \#7696.

10. K. H. Hofmann and P. Mostert, Splitting in topological groups, Mem. Amer. Math. Soc. No. 43 (1963). MR 27 \#1529.

11. J. F. Jakobsen, and W. Utz, The non-existence of expansive homeomorphisms on a closed 2-cell, Pacific J. Math. 10 (1960), 1319-1321. MR 22 \#8488.

12. L. S. Pontrjagin, Topological groups, 2nd ed., GITTL, Moscow, 1954; English transl. Gordon \& Breach, New York, 1966. MR 17, 171; MR 34 \#1439.

13. W. Reddy, The existence of expansive homeomorphisms on manifolds, Duke Math. J. 32 (1965), 627-632. MR 32 \#4679.

14. Ju. M. Smirnov, On metrization of topological spaces, Uspehi Mat. Nauk 6 (1951), no. 6 (46), 100-111; English transl., Amer. Math. Soc. Transl. (1) 8 (1962), 62-77. MR 14, 70.

15. W. Utz, Unstable homeomorphisms, Proc. Amer. Math. Soc. 1 (1950), 769-774. MR 12, 344.

16. R. F. Williams, A note on unstable homeomorphisms, Proc. Amer. Math. Soc. 6 (1955), 308-309. MR 16, 846.

17. T. S. Wu, Expansive automorphisms in compact groups, Math. Scand. 18 (1966), 23-24. MR 33 \#2759.

UNIVERSITY OF MisSOURI-COLUMBIA, Columbia, Missouri 65201 